International Journal of Engineering \& Technology, 7 (4) (2018) 2546-2551
International Journal of Engineering \& Technology
SPC
Website: www.sciencepubco.com/index.php/IJET
doi: $10.14419 /$ ijet.v7i4.14809
Research paper

\title{
Glaucoma diagnosis using discrete wavelet transform and histogram features from fundus images
}

\author{
Bhupendra Singh Kirar ${ }^{1}$, Dheeraj Kumar Agrawal ${ }^{2}$ \\ ${ }^{I}$ Ph.D. Research Scholar, Department of Electronics and Communication Engineering, Maulana Azad National Institute of Technology, \\ Bhopal, 462003, India \\ ${ }^{2}$ Assistant Professor, Department of Electronics and Communication Engineering, Maulana Azad National Institute of Technology, \\ Bhopal, 462003, India \\ *Corresponding author E-mail:bhup17@gmail.com
}

\begin{abstract}
Glaucoma is one of the main eye diseases; it cause progressive deterioration of optic nerve fibers due to increased fluid pressure. The existing methods of glaucoma diagnosis are time consuming, expensive and require practiced clinicians to understand the eye problems. Hence fast, cheap and more accurate glaucoma diagnosis methods are needed. This paper presents an innovative idea for diagnosis of glaucoma using third level two dimensional discrete wavelet transform (2D DWT) and histogram features from fundus images. The 2D DWT is used to decompose the glaucoma and healthy images and histogram features are extracted from 2D DWT decomposed sub band images. The least square support vector machine (LS-SVM) is used as a classifier which classifies the glaucoma and healthy images using the extracted features. The proposed method yielded classification accuracy of $88.33 \%, 87.50 \%$, and $86.67 \%$ for ten, eight and fivefold cross validation respectively. The obtained classification accuracy, sensitivity and specificity are $88.33 \%, 90.00 \%$, and $85.00 \%$ for tenfold cross validation respectively. Obtained results prove that the performance of the proposed method is better compared to the existing methods. It may considerably increases the diagnosis speed of ophthalmologists.
\end{abstract}

Keywords: Discrete Wavelet Transform; Feature Extraction; Glaucoma; Support Vector Machine; Pre-Processing.

\section{Introduction}

Glaucoma is a category of eye disease. It is the principal reason for the permanent blindness in the present world. It may lead to permanent loss of eye vision. It causes progressive deterioration of optic nerve fibers due to increased intraocular pressure (IOP) in the eyes [1]. It is expected that 64.3 million people suffered from glaucoma worldwide with age range of 40 to 80 (Years) in the year 2013 [2]. The typical value of intraocular pressure for glaucoma imaging is more than $21 \mathrm{mmHg}$ [3]. Around $12.3 \%$ loss of sight cases reported due to glaucoma worldwide [4].

Glaucoma is broadly classified as primary open angle glaucoma and primary angle closure glaucoma [5]. The first one is also well known as chronic glaucoma. It is the principal form of glaucoma in which drainage canals are clogged and become ineffective. If it is not cared in the early stage, it may lead permanent blindness. The angle closure glaucoma is the uncommon form and it is also known as acute glaucoma [6].

In the current scenario there are many methods used to detect the glaucoma present in the eye. These methods are medicinal examination of optic nerve head $(\mathrm{ONH})$ [7]; structural and geometrical changes in optic cup and disc, and medical background of the respective family (genetics) [8], and eye inspection methods [9]. In this decade, new trends will give opportunities for researchers to understand risk factors, detection and diagnosis of glaucoma in the early stage [10].

Recently the use of computer made the glaucoma detection and classification easy and fast. Based on computers there are several methods available for the diagnosis of glaucoma [11]. It has made the testing of glaucoma simple.

Scanning Laser Ophthalmoscope [12], Optical Coherence Tomography [13] and Heidelberg Retina Tomography [14] are well known methods and have been widely used for glaucoma diagnosis. These methods are slow, costly. Therefore, fast and low cost methods are required for glaucoma diagnosis.

There are several advanced methods used for diagnosis of glaucoma. Yadav et al. [15] used texture feature extraction and classification. Kolar et al. [16] extracted power spectral features and fractal dimensions feature and fed to SVM classifier. Bock et al. [1] extracted feature from FFT and B-spline coefficients and used PCA and SVM classifier. Raja et al. 2013 [17] used complex wavelet transform and higher order spectra. Raja et al. 2015 [18] used wavelet packet decomposition (WPD) and entropy and energy features. Townsend et al. [19] used heidelberg retinal tomograph (HRT) 3 parameters, recursive partition and regression tree with SVM classifier. Kim et al. [20] extracted 7 features including fractal analysis and classified using SVM classifier. These methods need to enhance the performance of their system.

This paper presents an innovative idea for diagnosis of glaucoma using third level 2D DWT and histogram features extracted from the fundus images. The input images are pre-processed and fed to the third level 2D DWT. 2D DWT decomposes input images in to sub band images. Histogram features namely mean, variance, skewness, kurtosis, energy and entropy have been extracted from 2D DWT decomposed sub band images. These extracted features are given as input to the least square support vector machine (LSSVM) classifier with radial basis function (RBF) kernel. Accuracy, sensitivity and specificity have been calculated as the perfor- 
mance parameters for glaucoma diagnosis. The obtained results have been compared with the existing methods to prove the effectiveness of the proposed method.

The remaining part of the paper is planned as follows: images and tools with the proposed methodology are given in section 2 . Result and discussion is explained in section 3. Conclusion is explained in sections 4 .

\section{Proposed methodology}

Figure 1 shows the structure of the proposed methodology. It comprises of input image, pre-processing and green channel image extraction from input images, third level two dimensional discrete wavelet transform, histogram feature extraction and classification using LS-SVM.

The proposed method uses third level 2D DWT and histogram features. In this paper only green channel has been used for the extraction of features. Extracted features have been normalized and used by the LS-SVM classifier with RBF kernel. The proposed method is verified using three fold, fivefold, eight fold and tenfold cross validation.

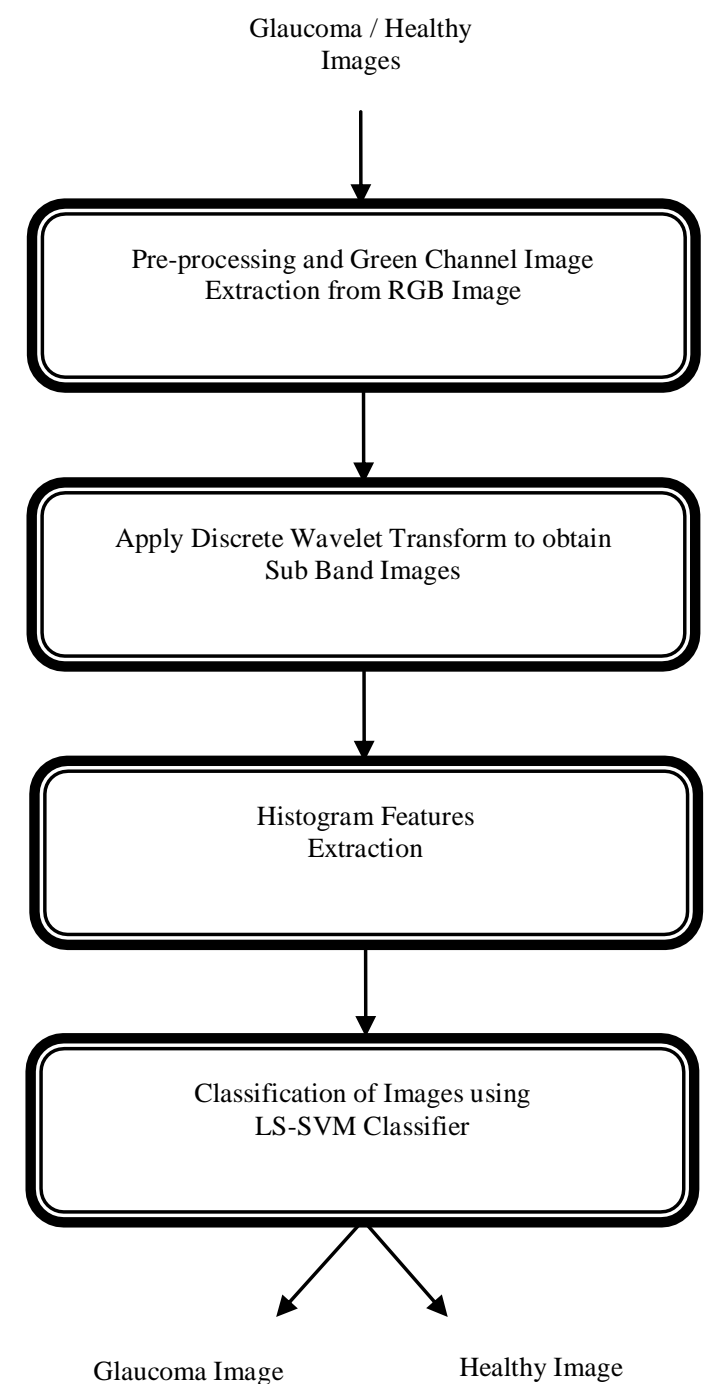

Fig. 1: Block Diagram of the Proposed Method.

\subsection{Images and tools}

For the proposed methodology the Rim1 image data set is used. It is publically available from the Medical Image Analysis Group (MIAG) [21]. In this paper 15 glaucoma and 15 healthy digital fundus images have been used. The proposed methodology is implemented using MATLAB at $2.10 \mathrm{GHz}$ with $2 \mathrm{~GB}$ RAM. Figure 2 shows the typical glaucoma and healthy digital fundus images.

(A)

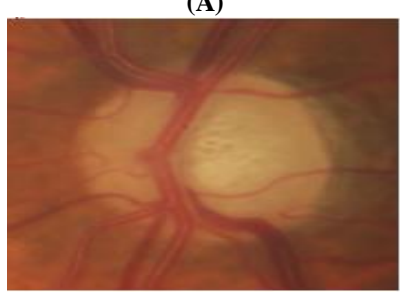

(B)

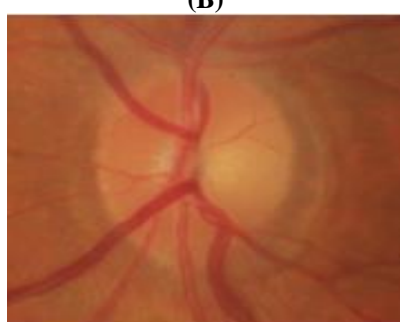

Fig. 2: Input Images (A): Glaucoma. (B): Healthy.

\subsection{Pre-processing}

The pre-processing plays an important role in image processing. Here input images are pre-processed. Firstly, the input images are resized to $256 \times 256$ to increase the processing speed and same resolution. Secondly, only green channel images are extracted from resized images as it contains more information [1]. Thirdly, green channel images are subjected to histogram equalization to increase contrast and dynamic range. Histogram equalization improves the appearance and contrast of image [22]. Lastly, these images are filtered to remove noise.

\subsection{Discrete wavelet transform}

It is very useful for time-frequency analysis of non-stationary signals. Discrete wavelet transform (DWT) is a very outstanding signal and image decomposition method [23]. It decomposes the input image into different sub band images. After each decomposition level, time resolution and frequency resolution is halved and doubled respectively [23]. It decomposes in to approximation coefficients and detailed coefficients. The approximation coefficients are further decomposed into approximation coefficients and detail coefficients for next level and the same procedure is repeated for three levels. The first sub-band image is the approximation coefficients. Second, third and fourth sub-band images are the detailed coefficients for the input images and these are also known as horizontal, vertical and diagonal coefficients respectively [24]. In this way different sub-band images are obtained using third level two dimensional discrete wavelet transform with dubechie wavelet (db4) from the input images. Third level two dimensional discrete wavelet transform is explained in [25].

The discrete wavelet transforms used discrete set of scale $(s=$ $\left.2^{-m}\right)$ and translation $\left(\tau=n 2^{-m}\right)$ parameters, where $\mathrm{m}$ and $\mathrm{n} \in U$, set of all integers. The family of wavelets is expressed as:

$\xi_{m, n}(t)=2^{\frac{m}{2}} \xi\left(2^{m} t-n\right)$

The decomposition of $x[n]$ signal on $J$ octave is expressed as:

$x[n]=\sum_{j=1 \text { to } J} \sum_{k \in U} c_{j, k} g\left[n-2^{j} k\right]+\sum_{k \in U} d_{J, k} h_{J}\left[n-2^{J} k\right]$

where $c_{j, k} j=1 \ldots J$ and $d_{j, k} j=1 \ldots J$ represents wavelet and scaling coefficients respectively and these are given as:

$c_{j, k}=\sum_{n} x[n] g_{j}^{*}\left[n-2^{j} k\right]$ 
$d_{j, k}=\sum_{n} x[n] h_{J}^{*}\left[n-2^{J} k\right]$

where, $\left(^{*}\right), g_{j}\left[n-2^{j} k\right]$ and $h_{J}\left[n-2^{J} k\right]$ represent complex conjugate, wavelet and scaling sequences, respectively.

The DWT approach towards images; DWT is implemented on each row and each column separately. Let $X$ denotes the image, and then the DWT decomposes the image in to approximation coefficient (low frequency) $X_{A}^{1}$, and details coefficients (high frequency) $X_{H}^{1}, X_{V}^{1}$ and $X_{D}^{1}$; corresponding to horizontal, vertical and diagonal coefficients. These four coefficients are known as sub band images and given as:

$X=X_{A}^{1}+\left(X_{H}^{1}+X_{V}^{1}+X_{D}^{1}\right)$

Second level decomposition is obtained by applying 2D DWT on $X_{A}^{1}$ (approximation coefficient). In this way $N^{t h}$ level decomposition can be obtained by applying 2D DWT on $N^{\text {th }}$ approximation coefficient $\left(X_{A}^{N}\right)$. The image $X$ can be written as:

$X=X_{A}^{N}+\sum_{i=1}^{N}\left(X_{H}^{i}+X_{V}^{i}+X_{D}^{i}\right)$

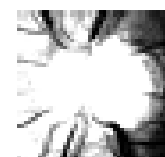

(A)

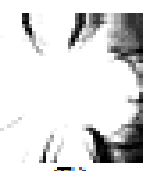

(E)

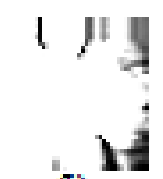

(I)

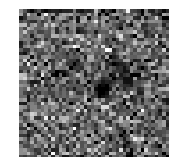

(B)

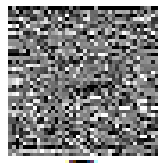

(F)

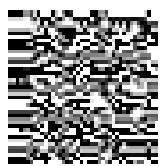

(J)

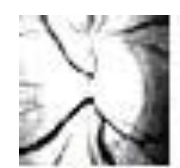

(A)

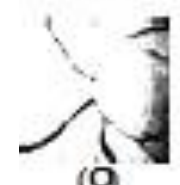

(Q)

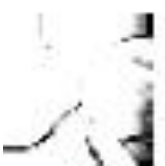

(U)

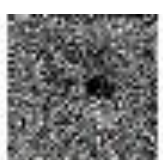

(N)

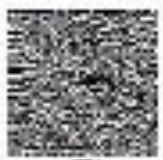

(R)

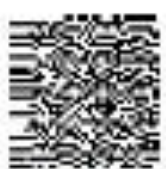

(V)

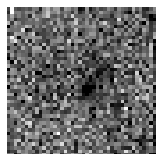

(C)

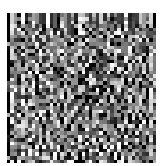

(G)

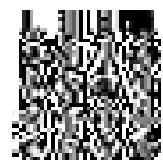

(E)

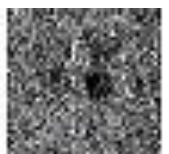

(O)

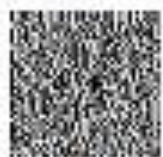

(3)

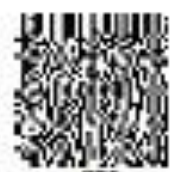

(W)

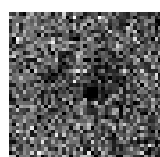

(D)

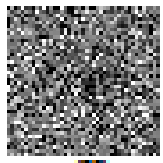

(H)

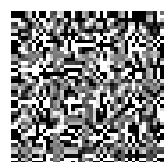

(L)

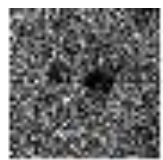

(P)

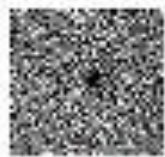

(T)

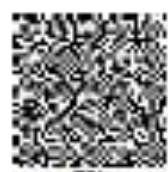

(X)
Fig. 3: Decomposed Sub Band Images Using 2D DWT. (A), (B), (C) and (D) are First Level, (E), (F), (G) and (H) are Second Level and (I), (J), (K) and (L) are Third Level of Decomposition of Glaucoma Image of Fig.2(A). (M), (N), (O) and (P) are First Level, (Q), (R), (S) and (T) are Second Level and (U), (V), (W) and (X) are Third Level of Decomposition of Healthy Image of Fig.2 (B).

Dubechie wavelet (db4) has been selected as mother wavelet because it renders outstanding classification accuracy. Decomposed sub band images using various decomposition level of 2D DWT for glaucoma and healthy images are shown in Figure 3.

\subsection{Feature extraction}

It is a very essential footstep for the classification of glaucoma. Most useful features have been used to capture the appropriate information from the images. In the proposed method various histogram features have been extracted from the decomposed sub band images. These are mean, variance, skewness, kurtosis, energy and entropy and given as follow:

\subsubsection{Mean}

It is defined as the measure of average intensity and denoted by $\mu$. It is given as [26], [27]:

$\operatorname{Mean}(\mu)=\sum_{i=0}^{L-1} y_{i} p\left(y_{i}\right)$

\subsubsection{Variance}

It is defined as the measure of the spread of value of $y$ about mean and denoted by $\sigma^{2}$. It is a measure of contrast and it is given as:

$\operatorname{Variance}\left(\sigma^{2}\right)=\sum_{i=0}^{L-1}\left(y_{i}-\mu\right)^{2} p\left(y_{i}\right)$

\subsubsection{Skewness}

It is defined as the measure of asymmetry of the data around the mean. It can be positive or negative, if the data are spread out more to the right or left respectively. It is zero for the symmetric distribution. It is a third moment, denoted by $\mu_{3}$ and is given as:

$\operatorname{Skewness}\left(\mu_{3}\right)=\sum_{i=0}^{L-1}\left(y_{i}-\mu\right)^{3} p\left(y_{i}\right)$

\subsubsection{Kurtosis}

It is defined as the measure of its relative flatness. It is a fourth moment and is denoted by $\mu_{4}$. It is given as:

$\operatorname{Kurtosis}\left(\mu_{4}\right)=\sum_{i=0}^{L-1}\left(y_{i}-\mu\right)^{4} p\left(y_{i}\right)$

\subsubsection{Energy}

It is defined as the measure of uniformity in the range [0, 1]. It is one for constant image. It is maximum when all intensity values are equal. It is denoted by $E_{g}$ and is given as:

$\operatorname{Energy}\left(E_{g}\right)=\sum_{i=0}^{L-1} p^{2}\left(y_{i}\right)$

\subsubsection{Entropy}

It is defined as the measure of uncertainty and randomness. Equal distribution of pixels intensity values leads to no information and hence zero entropy. The larger entropy will have more information and vice versa. It is denoted by $E_{n}$ and is given as [28]:

$\operatorname{Entropy}\left(E_{n}\right)=-\sum_{i=0}^{L-1} p\left(y_{i}\right) \log _{2} p\left(y_{i}\right)$

where, $p\left(y_{i}\right)$ is defined as the normalized histogram of the $y_{i}$ and $L$ is the number of grey level.

\subsection{Classification}

The proposed method used a least squares support vector machine (LS-SVM) classifier [29] with RBF kernel [30]. It is widely used to classify two or more classes. The use of LS-SVM in medical image classification is increasing regularly because it improved the performance of classification and helped medical experts for the diagnosis of glaucoma or other diseases. In LS-SVM hyperplanes are used to create a decision border line for the classification of classes. Suppose there are $N$ numbers of data points given as $\left\{p_{n}, q_{n}\right\}_{n=1}^{N}$, where $p_{n} \in \mathbb{R}^{m}$ is $n^{\text {th }}$ input data and $q_{n} \in \mathbb{R}^{m}$ represent class label for the corresponding $n^{\text {th }}$ data point. The LS- 
SVM discrimination function for two class classification is given as [29]:

$$
k(y)=\operatorname{sign}\left[\Omega^{T} z(p)+b\right]
$$

where $b$ represent bias, $\Omega$ represent weight vector of dimension $x$ and $z(p)$ is a function used to map $p$ in to $x$ dimensional space.

The decision function of LS-SVM classifier for two class classification is defined as [29]:

$k(y)=\operatorname{sign}\left[\sum_{n=1}^{N} a_{n} q_{n} K\left(p, p_{n}\right)+b\right]$

where $K\left(p, p_{n}\right)$ and $a_{n}$ represent the kernel function and Lagrange multiplier respectively.

Radial basis function (RBF) kernel is used which is given as [27]:

$K_{R B F}\left(p, p_{n}\right)=\exp \left[\frac{-\left\|p-p_{n}\right\|^{2}}{2 \sigma_{1}{ }^{2}}\right]$

where $\sigma$ is the width of RBF function.

\subsection{Performance parameters}

Performance parameters: Only three main performance parameters namely, sensitivity, specificity and accuracy have been used to evaluate the performance of the proposed method. These performance parameters are defined as [31]:

\subsubsection{Specificity}

It is a true negative rate (TNR). It is the ratio of correctly classified healthy images to the total healthy test images. It is calculated as:

$\operatorname{SPE}(\%)=\frac{T N}{T N+F P} \times 100$

\subsubsection{Sensitivity}

It is a true positive rate (TPR). It is the ratio of correctly classified glaucoma images to the total glaucoma test images. It is calculated as:

$\operatorname{SEN}(\%)=\frac{T P}{T P+F N} \times 100$

\subsubsection{Accuracy}

It is the ratio of fraction of correctly classified glaucoma and healthy images to the total number of test images. It is calculated as:

$A C C(\%)=\frac{T P+T N}{T P+T N+F P+F N} \times 100$

where $T P, T N, F P$ and $F N$ are defined as:

TP: True Positive, it is glaucoma images classified as glaucoma images.

$T N$ : True Negative, it is healthy images classified as healthy images.

$F P$ : False Positive, it is healthy images classified as glaucoma images.

$F N$ : False Negative, it is Glaucoma images classified as healthy images.

\section{Result and discussion}

The proposed method used third level 2D DWT and histogram features for glaucoma diagnosis. In the proposed methodology, the green channel images are extracted from the input fundus images and are decomposed by third level 2D DWT. Various histogram features namely mean, variance, skewness, kurtosis, energy and entropy have been extracted from the decomposed sub band imag- es. The LS-SVM with RBF kernel used the normalized features for the classification. Only three main performance parameters namely, sensitivity (SEN), specificity (SPE) and accuracy (ACC) have been calculated [30]. Table 1 shows the performance of proposed method using LS-SVM classifier with RBF kernel parameters (KP) and cross validation (CV) techniques for glaucoma diagnosis.

Table 1: Performance of Proposed Method for Glaucoma Diagnosis

\begin{tabular}{lllll}
\hline CV & KP & ACC $(\%)$ & SEN $(\%)$ & SPE (\%) \\
\hline 3 & 1.8 & 86.67 & 86.67 & 86.67 \\
5 & 1.7 & 86.67 & 86.67 & 86.67 \\
8 & 1.7 & 87.50 & 87.50 & 87.50 \\
10 & 1.8 & 88.33 & 90.00 & 85.00 \\
\hline
\end{tabular}

The obtained classification accuracies are $88.33 \%, 87.50 \%$, and $86.67 \%$ for ten, eight and fivefold cross validation with kernel parameter (KP) 1.8, 1.7 and 1.7 respectively [32]. The obtained classification accuracy, sensitivity and specificity are $88.33 \%$, $90.00 \%$ and $85.00 \%$ for tenfold cross validation respectively.

The kernel parameter value is varied from 0.1 to 2 with a step size of 0.1 . Figure 4 shows graph for accuracy versus kernel parameter for three, five, eight and tenfold cross validation. Figure 5, 6, 7 and 8 show plot for performance parameters versus kernel parameter for three, five, eight and tenfold cross validation respectively.

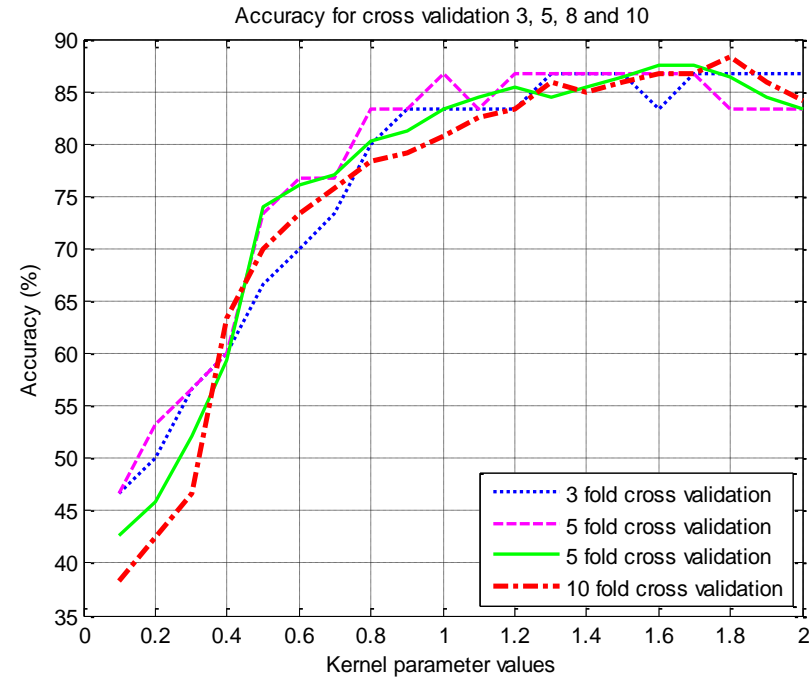

Fig. 4: Graph for Accuracy Versus Kernel Parameter Values for Three, Five and Ten Fold Cross Validation.

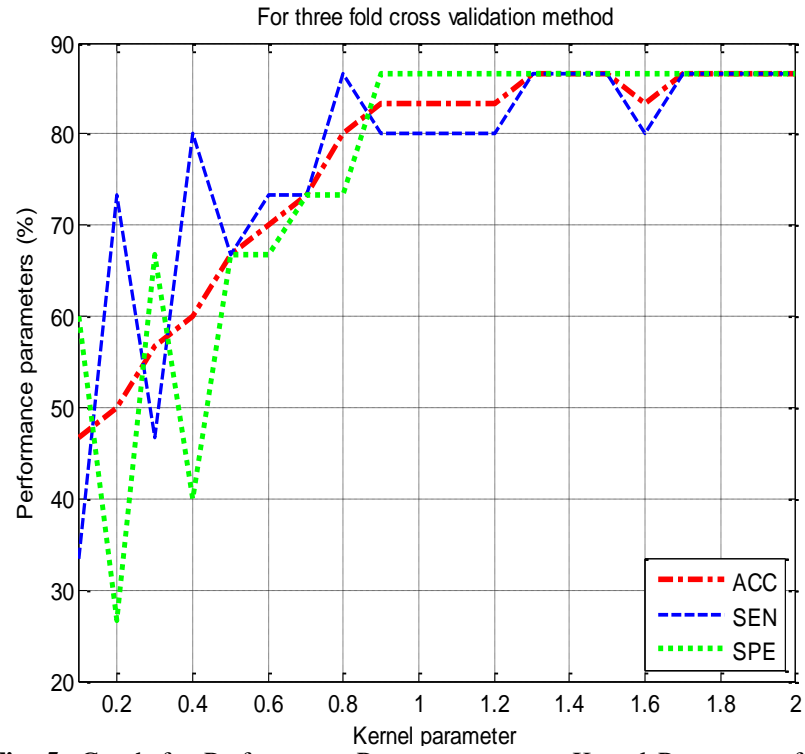

Fig. 5: Graph for Performance Parameters versus Kernel Parameters for Three Folds Cross Validation. 


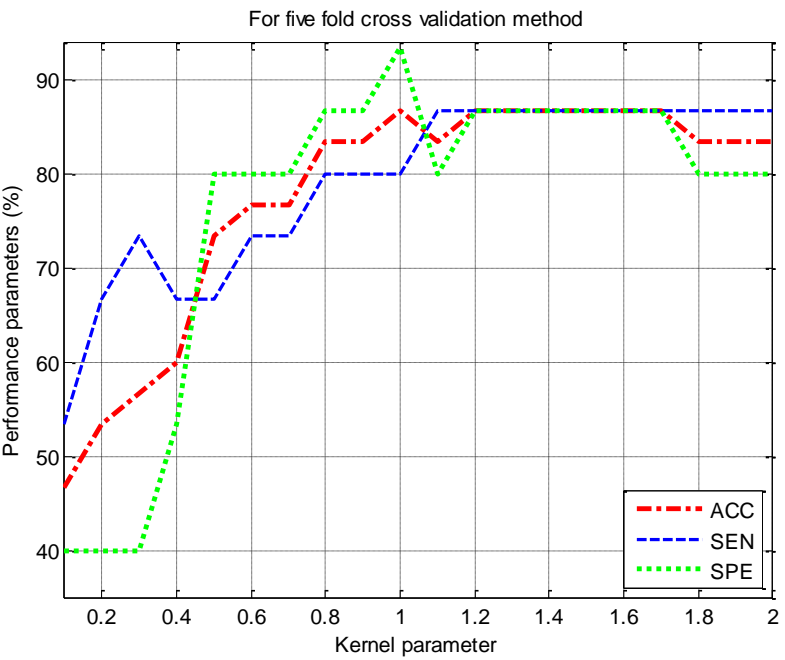

Fig. 6: Graph for Performance Parameters versus Kernel Parameters for Five Folds Cross Validation.

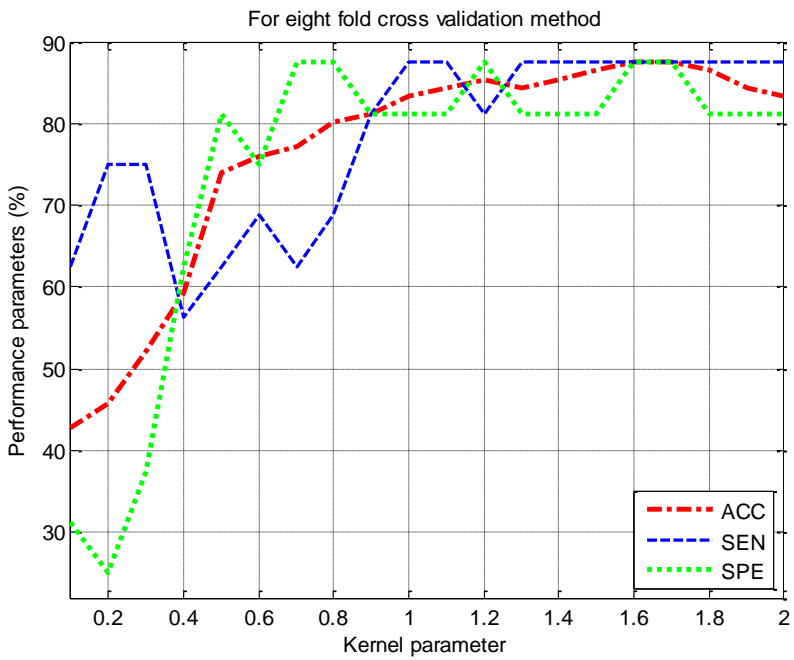

Fig. 7: Graph for Performance Parameters versus Kernel Parameters for Eight Folds Cross Validation.

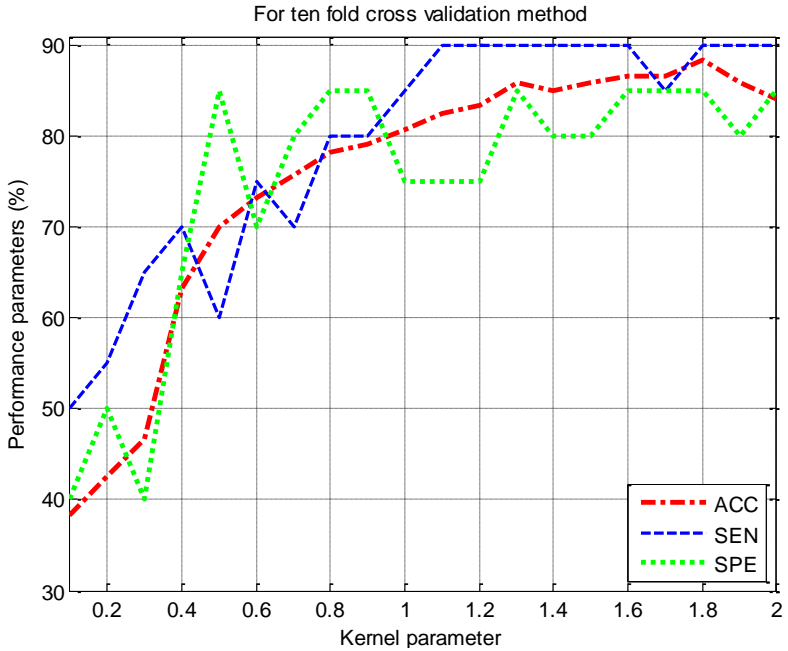

Fig. 8: Graph for Performance Parameters versus Kernel Parameters for Ten Folds Cross Validation.

The comparison of proposed and existing methods for glaucoma diagnosis is given in Table 2. Figure 9 shows the graph for comparison of methods for ten folds cross validation. Yadav et al. [15] used texture feature extraction and classification. They reported the classification accuracy of $72 \%$. Kolar et al. [16] explain about the retinal nerve fibre (RNF) of the glaucoma. It is characterized by regular damage. This method used two types of features namely, power spectral features and fractal features to analyse RNF.
This paper reported an accuracy of $74 \%$ using SVM classifier. Bock et al. [1] used intensity features extracted from B-spline and fast Fourier transform coefficients from pre-processed digital fundus images. The dimensionality of features is reduced by principal component analysis (PCA). This method reported the classification accuracy, sensitivity and specificity of $80 \%, 73 \%$ and $85 \%$ respectively using SVM classifier. Raja et al. 2013 [17] used complex wavelet transform and higher order spectra and reported the classification accuracy, specificity and sensitivity of $81 \%, 87 \%$ and $87 \%$ respectively. Raja et al. 2015 [18] used wavelet packet decomposition (WPD) and entropy and energy features are extracted. They reported the classification accuracy, sensitivity and specificity of $85 \%, 82 \%$ and $100 \%$ respectively. Townsend et al. [19] used heidelberg retinal tomograph (HRT) 3 parameters, recursive partition and regression tree with SVM classifier. They reported classification accuracy of $87.5 \%$. Kim et al. [20] extracted 7 features including fractal analysis and classified using SVM classifier. They reported the classification accuracy, sensitivity and specificity of $88 \%, 79 \%$ and $86 \%$ respectively.

In this paper the obtained classification accuracies are $88.33 \%$, $87.50 \%$, and $86.67 \%$ for ten, eight and fivefold cross validation with kernel parameter $1.8,1.7$ and 1.7 respectively [32]. The obtained classification accuracy, sensitivity and specificity are $88.33 \%, 90.00 \%$, and $85.00 \%$ for tenfold cross validation respectively.

Obtained results prove that the performance of the proposed method is better compared to the existing methods.

Table 2: Comparison of Proposed and Existing Methods

\begin{tabular}{llll}
\hline $\begin{array}{l}\text { Authors and } \\
\text { References }\end{array}$ & $\begin{array}{l}\text { Accuracy } \\
\text { (ACC) }(\%)\end{array}$ & $\begin{array}{l}\text { Sensitivity } \\
(\text { SEN) }(\%)\end{array}$ & $\begin{array}{l}\text { Specificity } \\
\text { (SPE) }(\%)\end{array}$ \\
\hline Bock et al. [1] & 80 & 73 & 85 \\
Yadav et al. [15] & 72 & NR & NR \\
Kolar et al. [16] & 74 & NR & NR \\
Raja et al. [17] & 81 & 87 & 87 \\
Raja et al. [18] & 85 & 82 & 100 \\
Townsend et al. [19] & 87.5 & NR & NR \\
Kim et al.[20] & 88 & 79 & 86 \\
Proposed Method & 88.3 & 90 & 85 \\
\hline
\end{tabular}

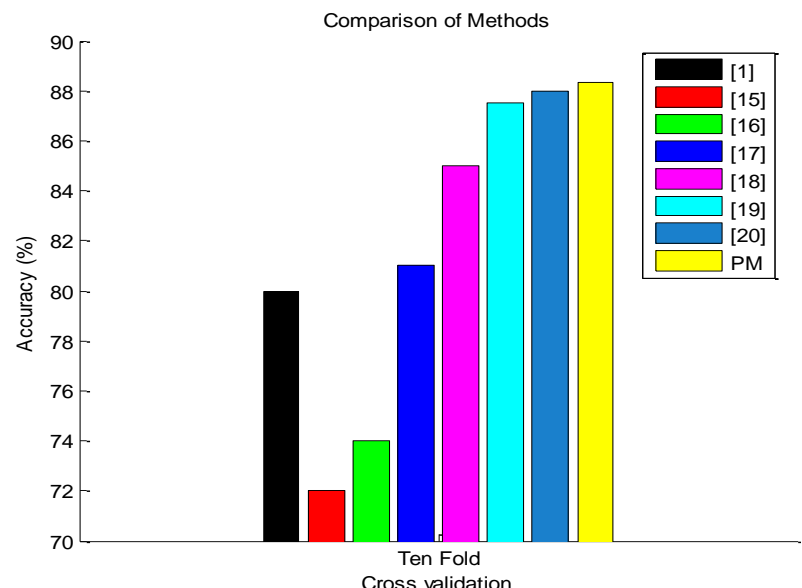

Fig. 9: Graph of Accuracy for Comparison of Existing Methods with Proposed Method (PM) for Ten Folds Cross Validation.

\section{Conclusion}

This paper presented a proposed method of glaucoma diagnosis using third level 2D DWT and histogram features using LS-SVM classifier with RBF kernel.

The green channel images are extracted from the digital fundus images and are decomposed using 2D DWT. Various histogram features have been extracted from the 2D DWT decomposed sub band images. The LS-SVM with RBF kernel used these features for the classification. The obtained performance parameters are given in Table 1. Only three main performance parameters namely, sensitivity, specificity and accuracy have been calculated. The 
obtained classification accuracies are $88.33 \%, 87.50 \%$, and $86.67 \%$ for ten, eight and fivefold cross validation with kernel parameter 1.8, 1.7 and 1.7 respectively. The obtained classification accuracy, sensitivity and specificity are $88.33 \%, 90.00 \%$, and $85.00 \%$ for tenfold cross validation respectively.

The proposed method was found to be effective in diagnosis of glaucoma and, with some continuous training, it could facilitate to the technicians and doctors to understand the eye disease fast and accurate. It may considerably increase the diagnosis speed of ophthalmologists.

The proposed methodology requires testing for huge database. The same approach can be used to extract other features from other diseases like ovarian cancer, fatty liver, diabetes and retinopathy.

\section{Acknowledgement}

Authors want to thank to Medical Image Analysis Group (MIAG) [21] for providing healthy and glaucoma digital fundus image database.

\section{References}

[1] Bock R, Meier J, Ny'ul LG, Hornegger J, \& Michelson G, "Glaucoma risk index: Atomated glaucoma detection from color fundus images", Medical Image Analysis, Vol. 14, No. 3, (2010), pp.471481.https://doi.org/10.1016/j.media.2009.12.006

[2] Tham YC, Li X, Wong TY, Quigley HA, Aung T \& Cheng CY, "Global prevalence of glaucoma and projections of glaucoma burden through 2040: A systematic review and meta-analysis", Ophthalmology, Vol. 121, No.11, (2014), pp. 20812090.https://doi.org/10.1016/j.ophtha.2014.05.013.

[3] Shen SY, Wong TY, Foster PJ, Loo JL, Rosman M, Loon SC, Wong WL, Saw SM \& Aung T, "The prevalence and types of glaucoma in Malay people: The Singapore Malay eye study", Investigative Ophthalmology and Visual Science, Vol. 49, No. 9, (2008), pp. 3846-3851.https://doi.org/10.1167/iovs.08-1759.

[4] Resnikoff S, Pascolini D, Etya'ale D, Kocur I, Pararajasegaram R , Pokharel GP \&Mariotti SP, "Global data on visual impairment in the year 2002", Bulletin of the World Health Organization, Vol. 82 , No.11, (2004)

[5] Nayak J, Acharya UR, Bhat PS, Shetty A \& Lim TC, "Automated diagnosis of glaucoma using digital fundus images", J. Med. Syst., Vol. 33, No. 5, (2009), pp. $337-$ 346.https://doi.org/10.1007/s10916-008-9195-z.

[6] Types of glaucoma, available online: https://www.glaucoma.org/glaucoma/types-of-glaucoma.php, last visit: 08.01.2017.

[7] Kavitha S, Zebardast N, Palaniswamy K, Wojciechowski R, Chan ES, Friedman DS, Venkatesh R \&Ramulu PY, "Family history is a strong risk factor for prevalent angle closure in a south Indian population", Ophthalmology, Vol. 121, No. 11, (2014), pp. 20912097.https://doi.org/10.1016/j.ophtha.2014.05.001

[8] Greaney MJ, Hoffman DC, Garway-Heath DF, Nakla M, Coleman $\mathrm{AL}$, \&Caprioli J , "Comparison of optic nerve imaging methods to distinguish normal eyes from those with glaucoma", Investigative Ophthalmology and Visual Science, Vol. 43, No. 1, (2002), pp. 140-145.

[9] Detecting glaucoma, available online: http://www.visionaware.org/info/your-eyecondition/glaucoma/detecting-glaucoma/125, last visit: 08.01.2017.

[10] Kersey Thomas, Clement Colin I, Bloom Phillip \&Cordeiro M. Francesca, "New trends in glaucoma risk, diagnosis \& management", Indian J Med Res., Vol. 137, (2013), pp 659-668.

[11] Song X, Song K \& Chen Y, "A computer-based diagnosis system for early glaucoma screening", Proceedings of the 27th Annual IEEE Engineering in Medicine and Biology, (2005), pp. 66086611 .

[12] Dua S, Acharya UR, Chowriappa P \&Sree VS, "Wavelet based energy features for glaucomatous image classification", IEEE Transactions on Information Technology in Biomedicine, Vol. 16, No. 1 , (2012), pp. 80-87.https://doi.org/10.1109/TITB.2011.2176540.

[13] Lim TC, Chattopadhyay S \& Acharya UR, "A survey and comparative study on the instruments for glaucoma detection", Medical Engineering \& Physics, Vol. 34, No. 2, (2012), pp.129. 139.https://doi.org/10.1016/j.medengphy.2011.07.030.
[14] Hermann MM, David F, Garway-Heath DF, Jonescu- Cuypers CP, Reinhard OW, Burk ROW, Jost B, Jonas JB, Mardin CY, Funk J \&Diestelhorst M, "Interobserver variability in confocal optic nerve analysis (HRT)," Int. Ophthalmol., Vol. 26, No. 4, (2005), pp.5143-149.

[15] Yadav D, Sarathi MP \& Dutta MK, "Classification of glaucoma based on texture features using neural networks", in IEEE 7th International Conference on Contemporary Computing, 2014.

[16] Kolar R \& Jan J, "Detection of glaucomatous eye via color fundus images using fractal dimensions", Radio Engineering, Vol. 17, No. 3, (2008), pp. 109-114.

[17] Raja C \&Gangatharan N, "Glaucoma detection in fundal retinal images using trispectrum and complex wavelet based features", European Journal of Scientific Research, Vol. 97 No. 1, (2013), pp. 159-171.

[18] Raja C \&Gangatharan N, "Appropriate sub-band selection in wavelet packet decomposition for automated glaucoma diagnoses", International Journal of Automation and Computing, Vol. 12, No. 4 , (2015), pp. 393-401.https://doi.org/10.1007/s11633-014-0858-6.

[19] Townsend KA, Wollstein G, Danks D, Sung KR, Ishikawa H, Kagemann L, Gabriele ML \&SchumanJS, "Heidelberg retina tomograph 3 machine learning classifiers for glaucoma detection", $B r . J$. Ophthalmol., Vol. 92, No. 6, (2008), pp. 814-818. https://doi.org/10.1136/bjo.2007.133074.

[20] Kim PY, Khan M. Iftekharuddin, Davey PG, M. T., Garas Anita, Hollo G, \&Essock EA, "Novel fractal feature-based multiclass glaucoma detection and progression prediction", IEEE Journal Of Biomedical And Health Informatics, Vol. 17, No.2, (2013), pp.269. 276.

[21] RIM1 Image dataset of Medical Image Analysis Group, available onlinehttp://medimrg.webs.ull.es, last visit: 28.07.2017.https://doi.org/10.1109/TITB.2012.2218661

[22] Hassan Rohayanti, KasimShahreen, Zubaidah WA, Wan ChekJafery, Shah ZA, "Image enhancement technique at different distance for iris recognition", International Journal on Advanced Science, Engineering and Information Technology, Vol.7, (2017), pp. 4-2.

[23] AlickovicEmina, Kevric Jasmin \&SubasiAbdulhamit, "Performance evaluation of empirical mode decomposition, discrete wavelet transform, and wavelet packed decomposition for automated epileptic seizure detection and prediction", Biomedical Signal Processing and Control, Vol. 39, (2018), pp. 94102.https://doi.org/10.1016/j.bspc.2017.07.022.

[24] Lahmiri Salim \&BoukadoumMounir "Biomedical image denoising using variational mode decomposition", Institute of Electrical and Electronics Engineers, Oct. 2014.

[25] Chaplot S, Patnaik LM, Jagannathan NR, "Classification of magnetic resonance brain images using wavelets as input to support vector machine and neural network", Biomed Signal Process Control, Vol. 1, No,1, (2006), pp. 8692.https://doi.org/10.1016/j.bspc.2006.05.002

[26] Gonzalez RC \& Woods RE, Digital image processing, Pearson, 2014.

[27] Gonzalez RC, Woods RE \&Eddins SL, Digital image processing Using Matlab, TMH Pvt. Ltd., 2010.

[28] Kirar BS and Agrawal DK, "Empirical wavelet transform based preprocessing and entropy feature extraction from glaucomatous digital fundus images", International Conference on Recent Innovations in Signal processing and Embedded Systems (RISE), Bhopal, 2017, pp. 315-319. https://doi.org/10.1109/RISE.2017.8378173.

[29] Suykens JAK and Vandewalle J, "Least squares support vector machine classifiers", Neural Processing Letters, Vol. 9, No. 3, (1999), pp. 293-300.https://doi.org/10.1023/A:1018628609742.

[30] Khandoker AH, Lai DTH, Begg RK, \&Palaniswami M, "Wavelet based feature extraction for support vector machines for screening balance impairments in the elderly", IEEE Transactions on Neural Systems and Rehabilitation Engineering, Vol. 15, No. 4, (2007), pp. 587-597https://doi.org/10.1109/TNSRE.2007.906961.

[31] SahuOmkishor, Anand V, KanhangadVivek\&Pachori RB, "Classification of magnetic resonance brain images using bi-dimensional empirical mode decomposition and autoregressive model", Biomed Eng Letter, Vol. 5, (2015), pp.311320.https://doi.org/10.1007/s13534-015-0208-9.

[32] Kohavi R, "A study of cross-validation and bootstrap for accuracy estimation and model selection", in International Joint Conference on Artificial intelligence, Vol. 14, No. 2, (1995), pp. 1137-1145. 